\title{
Interaction between molecular outflows and dense gas in the cluster-forming region OMC-2/FIR4
}

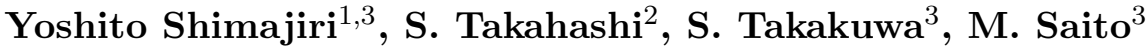 \\ and R. Kawabe ${ }^{3}$ \\ ${ }^{1}$ Department of Astronomy, School of Science, The University of Tokyo, Bunkyo, Tokyo \\ 113-0033,Japan \\ email:yoshito.shimajiri@nao.ac.jp \\ ${ }^{2}$ Department of Astronomical Science,Graduate Univercity for Advanced Studies, Osawa \\ 2-21-1, Mitaka, Tokyo 181-8588 Japan \\ ${ }^{3}$ ALMA-J project office, National Astronomical Observatory of Japan, Osawa 2-21-1, Mitaka, \\ Tokyo 181-8588 Japan
}

\begin{abstract}
Since most stars are born as members of clusters(Lada \& Lada 2003), it is important to clarified the detailed mechanism of cluster formation for comprehensive understanding of star formation. However, our current understanding of cluster formation is limited due to the followings;
\end{abstract}

(a)Cluster forming regions are located at the far distance.

(b) There are complex mixtures of outflows and dense gas in cluster forming regions.

So, we focused on the Orion Molecular Cloud 2 region (OMC-2), a famous cluster-forming region (Lada \& Lada 2003) and the most nearest GMC. We observed the FIR 4 region with the Nobeyama Millimeter Array(NMA), Atacama Submillimeter Telescope Experiment (ASTE). In this region, there are 3 protostars (FIR3, FIR4, FIR5) which were identified as $1.3 \mathrm{~mm}$ dust continuum sources (Chini et al. 1997) and driving sources of mixed outflows, and FIR 4 is the most strongest source of $1.3 \mathrm{~mm}$ dust continuum in OMC-2. Molecular lines we adopted are a high density $\left(10^{5} \mathrm{~cm}^{-3}\right)$ gas tracer of $\mathrm{H}^{13} \mathrm{CO}^{+}(\mathrm{J}=1-0)$, a molecular outflow tracer of ${ }^{12} \mathrm{CO}(\mathrm{J}=1-$ $0)$ and ${ }^{12} \mathrm{CO}(\mathrm{J}=3-2)$, and $\mathrm{SiO}(\mathrm{J}=2-1 \mathrm{v}=0)$ as a tracer of shocks associated with an interaction between outflows and dense gas.

From results of the ${ }^{12} \mathrm{CO}(\mathrm{J}=1-0)$ outflow, $\mathrm{H}^{13} \mathrm{CO}^{+}$dense gas, and the $\mathrm{SiO}$ shock, the outflow from FIR 3 interacts with dense gas in the FIR 4 region. Moreover the Position-Velocity diagram along the major axis of the ${ }^{12} \mathrm{CO}(\mathrm{J}=3-2)$ outflow shows that the ${ }^{12} \mathrm{CO}(\mathrm{J}=1-0)$ and $\mathrm{SiO}$ emission exhibits a $\mathrm{L}$ shape (the line widths increase in the interacting region in morphology). This is an evidence of interaction between the outflows and dense gas (Takakuwa et al. 2003). From result of the $3 \mathrm{~mm}$ dust continuum, the interacted region by the molecular outflow of FIR 3 is an assemble of seven dense cores. The mass of each core is $0.1-0.8 \mathrm{M}_{\odot}$. This clumpy structure is evident only at FIR 4 in the entire OMC-2/3 region. There are possible that two cores are in the proto-stellar phase, because $3 \mathrm{~mm}$ dust continuum source correspond to NIR source or $3.6 \mathrm{~cm} \mathrm{f}-\mathrm{f}$ jet source. From these results, cores in the FIR 4 region may be potential source of the next-generation stars. In the other words, there is a possibility that the molecular outflow ejected from FIR 3 is triggering the cluster formation in the FIR 4 region.

Keywords. OMC-2, molecular outflows, interaction 